\title{
Aportes conceptuales y epistemológicos en la producción escrita de Evelyn Levy al campo psicopedagógico: Interpelación interdisciplinaria y viscosidad del pensamiento
}

\author{
Ana Clara Ventura ${ }^{1}$ y Sandra Bertoldi ${ }^{2}$
}

\begin{abstract}
RESUMEN
Indagar en la producción de conceptos psicopedagógicos es altamente necesario para contribuir a una sólida relación entre episteme y profesión. A pesar de su notable importancia, hallamos escasos acuerdos internacionales en torno a cuáles son los conocimientos específicamente psicopedagógicos. En el marco de una investigación epistemológica más amplia, analizamos los aportes conceptuales y epistemológicos de la producción de Evelyn Levy al campo psicopedagógico. Construimos una técnica de análisis de contenido del discurso basada en cinco etapas: (1) Lectura cronológica; (2) Detección de conceptos teóricos/instrumentales y distinción entre denominación, definición y función interpretación; (3) Descripción del encuadre cultural de emergencia; (4) Análisis epistemológico de la presencia de desplazamientos teóricos, dinámica de continuidad (origen) y transformación (comienzo) conceptual; (5) Grado de rigurosidad epistemológica. Analizamos ocho artículos publicados entre los años 1982-2008, en revistas especializadas de Buenos Aires, y tres capitulos de libros, editados entre los años 1988-2001. Hallamos dos conceptos teóricos propios del campo psicopedagógico: interpelación interdisciplinaria y viscosidad del pensamiento, que muestran una actitud de vigilancia epistemológica al distanciarse de visiones normativas prescriptivas, pero que conservan el carácter constructivo de los conceptos de origen. Se discute la relevancia teórica y metodológica de este estudio para la construcción de un marco epistemológico específicamente psicopedagógico.
\end{abstract}

Palabras clave: investigación epistemológica, conceptos psicopedagógicos, práctica profesional, análisis de contenido de discurso, rigurosidad.

\section{Evelyn Levy's conceptual and epistemological contributions to the psychopedagogical field: Interdisciplinary interpellation and viscosity of thought}

\begin{abstract}
Inquiring the production of psychopedagogical concepts is highly necessary to contribute to a solid relationship between academic and professional field. Despite its remarkable importance, we find few international agreements on what specifically is psychopedagogical knowledge. Within the framework of a broader epistemological research, we analyze the conceptual and epistemological contributions of Evelyn Levy's production to the psychopedagogical field. We build a technique for the analysis of discourse content based on five steps: (1) Chronological reading; (2) Detection of theoretical / instrumental concepts and distinction between denomination, definition and function interpretation; (3) Description of the emergency cultural setting; (4) Epistemological analysis of the presence of theoretical displacements, continuity dynamics (origin) and conceptual transformation (beginning); (5) Degree of epistemological
\end{abstract} ${ }^{1}$ Consejo Nacional de Investigaciones Científcas y Técnicas (CONICET), Universidad Nacional del Comahue,
Argentina; ventura@comahue-conicet.gob.ar

2 Universidad Nacional del Comahue, Argentina. 
rigorousness. We analyzed eight articles published, between the years 1982-2008, in specialized journals of Buenos Aires and three book chapters, edited between the years 1988-2001. We found two theoretical concepts of the psychopedagogical field: interdisciplinary interpellation and viscosity of thought showing an attitude of epistemological vigilance by distancing themselves from prescriptive normative views, while preserving the constructive nature of the concepts of origin. The theoretical and methodological relevance of this study for the construction of a specifically psychopedagogical epistemological framework is discussed.

Keywords: epistemological research, psychopedagogical concepts, professional practice, discourse content analysis, rigorousness.

Desde su institucionalización académica, la Psicopedagogía en Argentina ha delimitado con mayor rigurosidad el perfil profesional de sus graduados que su consolidación e independencia epistémica respecto de otras disciplinas (Castorina, 2016; Giachetti \& Ventura, 2017). Los cuestionamientos y debates científicos son contados y dispersos por parte de los propios profesionales de la psicopedagogía, así como coexisten una diversidad de definiciones y posturas que han llevado a numerosos exponentes a problematizar su estatuto disciplinar (Artacho \& Ventura, 2011; Follari, 2013).

Actualmente, la Psicopedagogía se sitúa ante una doble problemática epistemológica: una externa o inter-disciplinar, la generación de fronteras más precisas respecto a sus campos fundacionales como la psicología y la pedagogía; y una problemática interna o intra-disciplinar, la construcción de mayores consensos en torno a qué, cómo y quiénes han producido conocimientos científicos en psicopedagogía en forma rigurosa y sistemática (Ortiz \& Mariño, 2014). De este modo, en este trabajo buscamos analizar conceptos y categorias específicamente psicopedagógicas que han surgido a nivel intra-disciplinar, distanciadas del sentido común y de la ligera 'importación' de términos y conceptos construidos por otras disciplinas.

En un sentido último, indagar acerca de la producción de categorias y conceptos específicos del campo psicopedagógico es altamente relevante para contribuir a una sólida relación entre episteme y profesión. Es más, es una tarea y responsabilidad de quienes la practican de hecho, es decir, de los propios psicopedagogos (Castorina, 2016; Follari, 2013).

A pesar de su notable importancia, hallamos escasas revisiones sistemáticas en la literatura en torno a la producción de conocimientos psicopedagógicos de referentes disciplinares que forjaron las bases de la psicopedagogía. Entre las excepciones, encontramos tres investigaciones.

Una fue elaborada en el marco del proyecto de investigación en curso "Producción de categorías en psicopedagogía y sus sustentos epistemológicos. Argentina 2014-2017" radicado en el Centro Universitario Regional Zona Atlántico de la Universidad Nacional del Comahue, dirigido por Bertoldi y codirigido por Enrico (Bertoldi \& Enrico, 2014-2017). Se analizan las contribuciones teóricas de ocho referentes nacionales, a saber: Alicia Fernández, Marina Muller, Clemencia Baraldi, Liliana González, Silvia Baeza, Norma Filidoro, Nélida Atrio y Evelyn Levy. Se puntualiza en la identificación de categorias y conceptos genuinos de cada referente, en el encuadre cultural de cada uno de ellos, los procedimientos racionales puestos en juego para su construcción y el grado de rigurosidad de los mismos. En este marco, dos artículos científicos dan cuenta de algunos resultados alcanzados. Por 
ejemplo, el artículo de Enrico y Fernández (2016) analiza las condiciones de aparición del concepto de inteligencia en la obra de Alicia Fernández. Las autoras identificaron que Fernández se nutre de la definición piagetiana de inteligencia. Las criticas que hace a las otras definiciones de inteligencia le sirven para ajustar la propia definición, para explicitar los supuestos y para dar cuenta de los efectos o consecuencias en el aprender: "la inteligencia permite al sujeto la inserción en la realidad y la invención de otras realidades posibles" (Fernández, 2000, p. 58). Si bien incorpora aportes y va desplegando nuevas ideas, las autoras consideran que mantiene el carácter constructivo, dinámico y orientado a la novedad de sus fundamentos de origen, sin contradecir el espíritu de las ideas piagetianas. No obstante, al pensar los problemas de aprendizaje desde la clínica psicopedagógica, necesariamente tiene que redefinir al sujeto e incorpora, junto con la dimensión lógica, la dimensión deseante. Por otro lado, Bertoldi y Tovani (2016) analizan el encuadre cultural de la obra de Liliana González. En este sentido, puede apreciarse como su formación académica transcurre desde una Psicopedagogía Reeducativa (sostenida en criterios epistemológicos de corte normativos) hacia una Psicopedagogía Clínica (criterios epistemológicos de corte reflexivo-crítico). A su vez, cómo su trayectoria profesional se va abriendo a distintas escenas (consultorio, cátedra, escuelas, talleres con padres y docentes, radio, televisión) y a considerar el impacto del entramado social en su trabajo clínico. Y cómo el nuevo contexto teórico-técnico que inaugura la clínica psicopedagógica, así como su posición reflexiva sobre su práctica, la lleva a reformular, desde la lectura psicoanalítica en su versión francesa, algunos conceptos, sin establecer una derivación lineal de la teoría a la práctica, sino considerando "los mecanismos de lectura, de interpretación sobre las prácticas que provee la teoria, para el conjunto de aplicaciones que podría dar lugar" (Bertoldi \& Tovani, 2016, p. 9). Así, despliega un tipo singular de aplicación, que hace funcionar la teoría de un modo novedoso e inédito, como por ejemplo en su consideración acerca de lo que es la clínica psicopedagógica y su concepto de aprendizaje y sus dificultades.

Una segunda investigación, integrada por docentes de la Licenciatura en Psicopedagogía de la Escuela de Humanidades de la Universidad Nacional de San Martin (sede Rosario), Giachetti y Ventura (2017) analizaron las contribuciones teóricas de Sara Pain a la especificidad del campo psicopedagógico. Para ello, exploraron su producción de categorías psicopedagógicas en su obra, así como determinaron su impacto en artículos científicos y profesionales publicados en cuatro revistas argentinas de psicopedagogía. Los resultados acerca de la producción conceptual indicaron que la noción transversal es aprendizaje, definido como un proceso que permite la transmisión del conocimiento hacia un sujeto en devenir. Respecto al impacto de su obra, se hallaron 10 artículos que citaron al menos una de las fuentes (respecto a los 662 analizados, esto es, 1,5\% del total). Las autoras interpretaron que, si bien la obra de Pain se considera un primer ensayo e impulso para los autores siguientes (por ejemplo, Alicia Fernández) en torno a la producción de categorias específicamente psicopedagógicas, los profesionales de dicho campo tendieron a nutrirse y recuperar en sus manuscritos referentes de áreas base del campo psicopedagógico tales como la psicología educacional, la pedagogía infantil o la neurologia.

La última investigación que hallamos, en el marco de una Tesis Doctoral en curso, se titula Las prácticas e investigaciones científicas en psicopedagogía en ámbitos universitarios público/estatales. Un análisis epistemológico en torno 
a los discursos y prácticas en la Argentina actual. En este trabajo, Bertoldi (2018) se propone reconstruir la conformación de espacios de producción de conocimiento psicopedagógico en seis universidades nacionales de Argentina (Universidad Nacional del Comahue, Universidad Nacional Patagonia Austral, Universidad Nacional de la Rioja, Universidad Nacional de Lomas de Zamora, Universidad Nacional de Rio Cuarto, Universidad Nacional de San Martín) que dictan la carrera en forma completa, identificar investigadores y/o equipos de investigación de referencia nacional y explorar bajo qué argumentos las definen como 'investigación psicopedagógica'. También indagar las condiciones, histórico-sociales y práctico-institucionales, de realización de las prácticas de investigación científicas, asî como analizar los procesos de investigación científica -según supuestos epistemológicos adoptados en los objetos construidos, en los enfoques conceptuales y metodológicos- y los productos científicos que se obtienen con el fin de establecer si crean argumentos coherentes y fructíferos para fundamentar científicamente a la disciplina. En una ponencia, Bertoldi (2017) se pregunta: ¿existe un campo científico específico? y responde que existe un campo científico en construcción. Y siguiendo los postulados de Bourdieu, lo argumenta por la existencia de un espacio de juego que lucha por bien, un capital, el saber psicopedagógico; por el volumen de los equipos de investigación (aproximadamente, 34), de sus trayectorias y de las investigaciones identificadas, porque hay productos y productores; por la presencia de una lucha competitiva entre los investigadores que tiene por desafio específico el monopolio de la autoridad científica (prestigio, reconocimiento, celebridad); por la existencia de un interés genérico del campo; por los diferentes estados del campo; por la existencia de instituciones de difusión/circulación de la producción científica.

Entre los referentes de la psicopedagogía en Argentina se sitúa Evelyn Levy, en el marco de la psicopedagogía terapéutica temprana. Levy obtiene su primer título universitario en la Universidad de Buenos Aires. Graduada como Doctora en Filosofia, se dedica rápidamente a la génesis del conocimiento. Adjunta a la enseñanza universitaria sobre Psicología de la inteligencia, una intensa actividad en el campo de la clínica de los problemas del aprendizaje la condujo a tornar al tema de cómo es posible comprender y aprender, y cómo era posible no comprender ni aprender. Esto es, cuáles son los obstáculos que impiden un pensamiento constructivo de conocimientos, dando lugar al concepto de "viscosidad del pensamiento" desde un posicionamiento epistemológico que ha denominado "interpelación interdisciplinaria".

\section{Objetivo general}

Analizar, desde un punto de vista epistemológico, los aportes conceptuales de la producción escrita de Evelyn Levy al campo psicopedagógico, poniendo el foco en dos conceptos originales de la autora: "interpelación interdisciplinaria" y "viscosidad del pensamiento", en términos de sus encuadres culturales, sus conceptualizaciones (denominación, definición y función interpretación), sus desplazamientos conceptuales y grado de rigurosidad de su producción escrita. 


\section{METODOLOGÍA}

\section{Diseño}

Se realizó un estudio bibliográfico de carácter teórico dirigido a la revisión, sistematización y análisis crítico de la producción escrita de la psicopedagoga Levy. Se desplegó una investigación de tipo teórica para seleccionar los conceptos producidos, sus condiciones y modos de producción, en el campo de la psicopedagogía. Para ello se construyó una técnica de indagación denominada herramienta de lectura epistemológica para analizar textos psicopedagógicos (Bertoldi \& Porto, 2015) que permitió analizar en detalle las producciones teóricas (libros, artículos científicos, conferencias) de los principales referentes nacionales.

\section{Corpus de estudio}

El corpus está integrado por dos tipos de fuentes primarias: libros y artículos científicos de autoría de Evelyn Levy. De su obra, accedimos a ocho artículos publicados, entre los años 1982-2008, en revistas especializadas de Buenos Aires y accedimos a tres capítulos de libros editados por Jerusalinsky y Castorina, entre los años 1988-2001, en Ediciones Nueva Visión y Eudeba, Buenos Aires.

\section{Procedimiento y análisis de datos}

Recuperando los lineamientos de análisis de la técnica construida para este estudio, el contenido del discurso escrito plasmado en las fuentes se analizó mediante las siguientes etapas: (1) Lectura cronológica de los textos localizados del autor; (2) Detección de los conceptos teóricos/instrumentales que el autor presenta como propios, distinguiendo su denominación, definición y función interpretación (Canguilhem, 2009); (3) Descripción del contexto de aparición/encuadre cultural en el que se forma el concepto; (4) Definición de la presencia o no de desplazamientos/préstamos teóricos de otros espacios intelectuales al nuestro, dinámica de continuidad (origen) y transformación (comienzo). De este modo, hace entrar al concepto, en palabras de Follari (2016), al menos, en tres relaciones: a.- saber tomar correctamente el concepto de otro campo disciplinar elegido por su pertinencia al campo psicopedagógico (mantiene su versión inicial); b.- hacer funcionar el concepto en una nueva situación no prevista en su versión inicial (una nueva aplicación); c.- producir un nuevo concepto (nueva relación entre definición y aplicación); (5) Grado de rigurosidad epistemológica.

\section{RESULTADOS}

Este apartado puntualiza la posición epistemológica en la producción escrita de Evelyn Levy y analiza el encuadre cultural; denominación, definición, función interpretación; desplazamiento conceptual de dos conceptos específicamente psicopedagógicos: Interpelación interdisciplinaria y viscosidad del pensamiento. Por último, se detalla el grado de rigurosidad epistemológica de ambos conceptos. 
Tabla 1.

El corpus de Evelyn Levy del presente estudio.

\begin{tabular}{|c|c|c|}
\hline Año & Artículos Científicos & Síntesis temática \\
\hline $\begin{array}{l}\text { Levy } \\
(1982)\end{array}$ & $\begin{array}{l}\text { Un enfoque psicopedagógico en el trabajo clínico } \\
\text { con niños deficientes mentales. Revista } \\
\text { Argentina de Psicología, 31, 57-72. }\end{array}$ & $\begin{array}{l}\text { Su posición psicopedagógica a partir de la } \\
\text { presentación de dos casos clínicos. }\end{array}$ \\
\hline $\begin{array}{l}\text { Levy } \\
(1987)\end{array}$ & $\begin{array}{l}\text { El psicopedagogo, la interdisciplina, el } \\
\text { conocimiento y el otro. Temas de } \\
\text { Psicopedagogía, 3, 109-124. }\end{array}$ & $\begin{array}{l}\text { Abordajes psicopedagógicos y psicomotrices } \\
\text { sobre la base de dos cuerpos teóricos: la } \\
\text { psicogénesis y el psicoanálisis. }\end{array}$ \\
\hline $\begin{array}{l}\text { Levy } \\
(1990)\end{array}$ & $\begin{array}{l}\text { Sujeto y objeto en la clínica psicopedagógica. Su } \\
\text { presencia y posición. Temas de Psicopedagogía, } \\
4,47-63 \text {. }\end{array}$ & $\begin{array}{l}\text { La no complementariedad del sujeto y el objeto en } \\
\text { la clínica psicopedagógica. }\end{array}$ \\
\hline $\begin{array}{l}\text { Levy } \\
(1991)\end{array}$ & $\begin{array}{l}\text { Posición e intervención en la dirección del } \\
\text { tratamiento psicopedagógico. Temas de } \\
\text { Psicopedagogía, 5, 43-57. }\end{array}$ & $\begin{array}{l}\text { Las estrategias terapéuticas en el tratamiento y } \\
\text { sus efectos. }\end{array}$ \\
\hline $\begin{array}{l}\text { Levy } \\
(1992)\end{array}$ & $\begin{array}{l}\text { El proceso diagnóstico en la intervención } \\
\text { psicopedagógica. Aprendizaje Hoy, 23/24, 7-13. }\end{array}$ & $\begin{array}{l}\text { Las modalidades diagnósticas de la clínica } \\
\text { psicopedagógica como consecuencia de una } \\
\text { posición específica del psicopedagogo respecto a } \\
\text { la práctica analítica, la fonoaudiología, la } \\
\text { neuropediatría y la psicomotricidad. }\end{array}$ \\
\hline $\begin{array}{l}\text { Levy } \\
(1994)\end{array}$ & $\begin{array}{l}\text { De la significación a la intervención en la clínica } \\
\text { psicopedagógica hospitalaria. Temas de } \\
\text { Psicopedagogía, } 6,195-205 \text {. }\end{array}$ & $\begin{array}{l}\text { La manera de hacer trabajar nuestros } \\
\text { interrogantes supone una toma de posición teórica } \\
\text { y clínica que, enlazada a aspectos éticos e } \\
\text { ideológicos, determina una particular relación } \\
\text { significación-intervención. }\end{array}$ \\
\hline $\begin{array}{l}\text { Levy } \\
(1995)\end{array}$ & $\begin{array}{l}\text { Viscosidad de pensamiento y procesos } \\
\text { cognoscitivos. Una interpelación interdisciplinaria. } \\
\text { Escritos de la Infancia, 6, 15-33. }\end{array}$ & $\begin{array}{l}\text { La viscosidad de pensamiento en los procesos } \\
\text { cognoscitivos es un observable clínico en niños } \\
\text { con patologías de desarrollo, así como en niños } \\
\text { normales. }\end{array}$ \\
\hline $\begin{array}{l}\text { Levy } \\
(1996)\end{array}$ & $\begin{array}{l}\text { A interpelação interdisciplinar na clínica } \\
\text { psicopedagógica. Estilos } D a \text { Clínica, 1(1), 128- } \\
\text { 145. }\end{array}$ & $\begin{array}{l}\text { La interpelación interdisciplinar es el foco del } \\
\text { trabajo. }\end{array}$ \\
\hline Año & Capítulos de libro & Síntesis temática \\
\hline $\begin{array}{l}\text { Levy } \\
\left(1988^{a}\right)\end{array}$ & $\begin{array}{l}\text { Reflexiones en torno a la clínica psicopedagógica } \\
\text { y psicomotriz (pp. 150-162) }\end{array}$ & $\begin{array}{l}\text { Es ilusorio pretender el dominio de la situación de } \\
\text { adquisición del conocimiento en la práctica } \\
\text { psicopedagógica y psicomotriz. }\end{array}$ \\
\hline $\begin{array}{l}\text { Levy } \\
\text { (1988b) }\end{array}$ & $\begin{array}{l}\text { Análisis de los procesos de construcción de la } \\
\text { lengua escrita en niños con síndrome de Down. } \\
\text { Abordaje psicogenético (pp. 265-288) }\end{array}$ & $\begin{array}{l}\text { Alfabetización y desarrollo intelectual de niños con } \\
\text { Síndrome de Down. }\end{array}$ \\
\hline $\begin{array}{l}\text { Levy } \\
(2001)\end{array}$ & $\begin{array}{l}\text { Posibles implicancias recíprocas entre Psicología } \\
\text { Genética y Clínica Psicopedagógica. Estrategias } \\
\text { y procedimientos clínicos (pp. 291-312) }\end{array}$ & $\begin{array}{l}\text { Observables e intervenciones en la clínica } \\
\text { psicopedagógica. }\end{array}$ \\
\hline
\end{tabular}

\section{Análisis de la posición epistemológica en la producción escrita de Evelyn Levy}

La posición epistemológica de la autora se construye sobre la base de una serie de interrogantes y problemáticas, tanto conceptuales como clínicas, de las prácticas psicopedagógicas en relación con otras prácticas terapéuticas que se ocupan del aprendizaje (e.g. fonoaudiología, psicología, neuropediatria, psicomotricidad). Cabe destacar que estas otras prácticas terapéuticas, a su vez, son referentes teóricos a los cuales recurren las prácticas 
psicopedagógicas para construir, en términos de la autora, su propio "andamiaje conceptual".

Entre las problemáticas conceptuales, la autora destaca una articulación imposible entre la teoría psicogenética y psicoanalítica en una teoría más abarcativa, debido a que remiten a sujetos teóricos diferentes. Entre las problemáticas clinicas, la autora expresa la presencia de "intervenciones fallidas" como evidencias manifiestas de las limitaciones e insuficiencias de dichos cuerpos teóricos para dar respuestas psicopedagógicas específicas acerca de ese sujeto de conocimiento (Levy, 2001). En palabras de la autora "cuando convocamos en diferentes momentos de nuestra conceptualización a dichos "saberes endiosados": ¿qué esperábamos? ¿acaso que desde sus conceptos y palabras la justeza de nuestra práctica con sus discursos se garantizara? (Levy, 1990, p. 47).

Los recortes técnicos, la selección y oferta de situaciones, no es ni azarosa ni ingenua y responde a una cierta manera de hacer trabajar la relación significación-intervención en la mirada y escucha psicopedagógica.

\begin{abstract}
Las preguntas por el sujeto social, inconsciente o epistémico, con sus singulares articulaciones, determinan, evidentemente, modalidades diferentes de intervención. La exclusión-omisión o suspensión de ciertas significaciones en la intervención, la yuxtaposición y/o repetición de conceptos extrapolados de otras disciplinas, sin mediatización, evidencian la problemática articulación de los diferentes discursos en relación con las dificultades de aprendizaje del sujeto. Si en algunos casos las centraciones en una u otra significación son el efecto inevitable de los momentos por los que transcurre la clínica y la formación en otros, parece definir una posición (Levy, 1994, pp. 196-197).
\end{abstract}

Por tanto, no solo los referentes teóricos encaminan la intervención y conceptualización, también los referentes clínicos tienen como efecto poner en movimiento ciertos recortes en el proceso de significación y conceptualización. Los distintos referentes teóricos a los que necesariamente el psicopedagogo se acerca dirigen los movimientos de su mirada y hacen que preste atención a un aspecto, quedando los otros inevitablemente borrados, omitidos-suspendidos, privilegiando otra significación en el intento de hacerla trabajar. Cuando desde los referentes teóricos se da cuenta de los fenómenos clínicos a los que se asiste privilegiando una u otra lectura, se encamina hacia un determinado rumbo la intervención. Por supuesto que no solo los referentes clínicos constituidos, sino los fenómenos clínicos a los que se asiste también inciden en la intervención.

En otros términos, "cuando asistimos a lecturas parciales en los que la intervención se piensa desde un único recorte, observamos operaciones de exclusión-omisión; las operaciones de sustracción, por efecto de una suspensión momentánea, suponen otra posición" (Levy, 1994, p. 200). Por el tipo de intervención clínica e institucional es como se pueden reconocer las diferentes significaciones.

\footnotetext{
Las operaciones de sustracción implican la suspensión en relación a algunas significaciones, suspensión momentánea e inevitable por efecto de centración en una significación particular. Diferente es la operación de exclusión-omisión: la exclusión de ciertas relaciones en la determinación a priori de algunos recortes técnicos, como las adscripciones y defensas ciegas por la verdad de uno de los discursos en cuestión, ponen de manifiesto estas operaciones de omisión-exclusión (Levy, 1994, p. 200).
}

Privilegiar discursos, ofertas, producciones en relación con múltiples significaciones, hace a la especificidad de una intervención y posición. Ciertamente es imposible, por estructura, abordar simultáneamente las múltiples lecturas en una única conceptualización. 
Interpelación interdisciplinaria: encuadre cultural; denominación, definición, función interpretación; desplazamiento conceptual

\section{Encuadre Cultural}

Históricamente, una buena parte de los intentos interdisciplinarios psicopedagógicos no llegaban a ser tales pues se reducian a disyunciones o sumatorias parciales, limitadas a duplas de recortes disciplinarios entre neurología y psicoanálisis, psicología genética y neurología o psicología genética y psicoanálisis. Las intervenciones en la práctica clínica, así como las conceptualizaciones, movilizaban distintas reflexiones disciplinarias que usualmente recorrían paralelamente el fenómeno clínico. Estas situaciones reflejaban ciertos grados de confusión entre abordajes multi e interdisciplinarios (Levy, 2001, p. 294).

En 1990, sin enunciar propiamente el término, Levy sienta sus bases fundacionales en una nota al pie meta-analítica explicitando que, históricamente, los psicopedagogos pensábamos el significado y el sentido del síntoma durante el proceso diagnóstico como hipótesis a confirmar. Con el tiempo fuimos descubriendo que, si pensábamos con relación a la legalidad del inconsciente, ya no podíamos sostener esa escucha y posición. Las formaciones del inconsciente no podían pensarse desde un saber ya sabido del terapeuta o desde una psicología comprensiva.

Ante esta problemática, Levy propone que no se trata de pivotear entre las conceptualizaciones de otras disciplinas para intentar aplicarlas a nuestra clínica. En el interior de la misma, los psicopedagogos fueron interpelando su propia intervención y su especificidad, intentando lograr una conceptualización mejor (Levy, 1990). Son reflexiones que acompañan nuestro pensar respecto al sujeto de conocimiento (...) en las perturbaciones que acontecen en el camino de su construcción cognitiva y en su proceso de aprendizaje" (Levy, 1987, p. 114). En este contexto, emerge el concepto de "interpelación interdisciplinaria" en el seno de la intervención psicopedagógica como una plataforma para tomar una posición teórica y clínica que, enlazada a aspectos éticos e ideológicos, determina una particular relación significación-intervención (Levy, 1994).

\section{Denominación, definición, función interpretación}

La denominación del concepto "interpelación interdisciplinaria" refiere a la forma de abordar los problemas de aprendizaje. Cuando desde una posición de autonomía los diferentes discursos dan cuenta de las dificultades de aprendizaje de un niño, lo hacen desde el interior de sus referentes teóricos, dentro de los márgenes de una particular significación intra-disciplinaria. Estas diferentes versiones acerca de dichas dificultades, al ser planteadas desde el exterior de la clínica psicopedagógica - y por lo tanto desconocer algunos de los problemas específicos de la misma - no pueden trasladarse directamente a nuestra intervención. En este sentido, las contribuciones conceptuales y clínicas no pueden considerarse agotadas y se hace necesario interpelar los fenómenos clínicos interdisciplinariamente.

La definición del concepto "interpelación interdisciplinaria" en la intervención psicopedagógica supone la puesta en acción de los tres movimientos teórico-clínicos involucrados en el proceso de interpelación interdisciplinaria en el interior del fenómeno clínico mismo (Levy, 1996). Esto es, en una primera instancia, un movimiento entre el exterior y el interior de 
la intervención psicopedagógica. En una segunda instancia, se trata de trabajar sobre las consecuencias de las indagaciones anteriores al interior de la propia intervención y conceptualización clínica. Un tercer movimiento se suscita cuando a partir de las reformulaciones y ampliaciones dadas previamente, es posible el relanzamiento de nuevas preguntas a las otras especialidades.

Por ejemplo, un niño con problemas en el desarrollo implica una serie de interpelaciones interdisciplinarias debido a la interpenetración entre el desarrollo, la constitución orgánica y la estructura del lenguaje que provoca una relación entre estos órdenes de anudamiento pero, a su vez, una interpelación y tensión que puede llegar a des-anudar lo ya anudado o impedir sucesivos anudamientos. La configuración clínica de un problema en el desarrollo de un niño es siempre una interpelación por los límites que la causan.

Por último, la función interpretación del concepto "interpelación interdisciplinaria" refiere a la posibilidad de enriquecer la clínica, ampliar las teorías de los campos de referencia y contribuir a la especificidad conceptual de las prácticas profesionales psicopedagógicas (Levy, 2001, pp. 294-295). El ámbito de inserción donde se desarrolla la práctica psicopedagógica determina ciertos recortes en la intervención. Esta será diferente si se realiza desde una única lectura, desde sus múltiples significaciones, que si se lleva a cabo desde una interpelación interdisciplinaria puesta en movimiento por un fenómeno clínico en sí mismo.

\section{Desplazamiento conceptual}

A lo largo del análisis de su producción escrita, es posible inferir que la autora recupera las tesis fundamentales de la obra freudiana y lacaniana para dar forma a la denominación, definición y función interpretación del concepto "interpelación interdisciplinar", otorgándole un sentido original al interior de las prácticas psicopedagógicas.

Desde una posición interdisciplinaria, Levy plantea que en la singular construcción de las dificultades de aprendizaje del sujeto se articulan de modo particular su constitución subjetiva, neurofuncional y cognitiva. Ellas determinan la posibilidad y modalidad de intercambio con otros y con los distintos objetos de conocimiento. Cuando se intenta dar cuenta de esa articulación en el análisis de las dificultades de aprendizaje, cuando se piensa las propuestas diagnósticas o las estrategias en la dirección de un tratamiento, hay que pensar acerca de cómo se ponen en movimiento esas diferentes significaciones.

Por lo expuesto, es posible interpretar que su aporte consiste en un proceso de reelaboración y resignificación de los conceptos de "interpelación" e "interdisciplina", articulándolos en un marco pragmático o de uso efectivo en un contexto históricamente situado como es el de las prácticas terapéuticas psicopedagógicas.

Por estos argumentos, ubicamos la 'interpelación interdisciplinaria' como un nuevo concepto teórico propio del campo psicopedagógico (Follari, 2016). 

Viscosidad del Pensamiento: encuadre cultural; denominación, definición,
función interpretación; desplazamiento conceptual

\section{Encuadre cultural}

Desde una interpelación interdisciplinaria de sus prácticas, la autora investigó la modalidad del pensamiento de niños en sus dimensiones cognoscitivas, libidinal y neurofuncional, respecto de cada objeto de conocimiento, en la singularidad del caso a caso. Para dar cuenta de las problemáticas que observaba en niños, apeló al concepto de "viscosidad del pensamiento".

La autora señala la necesidad de alternar un saber con un no saber (Levy, 1987). Resulta relevante reducir la influencia del imaginario, desprendiéndonos de una posición narcisista al colocar al niño en el lugar ideal del yo. Se hace necesario, pues, remitir al niño a otro objeto diferente de uno mismo. El psicoanálisis puede aportarnos en ese sentido una ética en la cual nuestras prácticas pueden inspirarse. Esta implica la desmitificación del lugar engañoso de poseedor de un saber y completud. Ética que se constituye en el respeto por la palabra del paciente, en la verdad de su discurso. Desde una comprensión de los postulados psicogenéticos, podríamos pensar en una ética en el sentido de la actitud que el terapeuta asuma frente al proceso del conocimiento del niño. A ambas éticas se refiere la autora cuando plantea alternar un saber con un no saber (Levy, 1987).

Su lectura acerca de esta adhesividad de pensamiento en la infancia está anclada en el concepto de viscosidad propuesto por Inhelder en sus investigaciones con niños con deficiencia mental. Planteó que, si bien avanzan en sus razonamientos, atravesando los mismos procesos que los niños normales, lo hacen a ritmos diferentes, con fijaciones en la elaboración de las operaciones y sus agrupamientos. En los niños deficientes hay una fijación y detenimiento a una estructura operatoria inacabada.

A su vez, esta lectura está inspirada en el concepto de viscosidad o fijación libidinal. Esto es, recuperó la noción freudiana de viscosidad libidinal, en el sentido de una inercia psíquica. Al extender este concepto al pensamiento, plantea el término de viscosidad del pensamiento o inercia mental. En otros términos, la hipótesis de la autora se alinea con la tesis freudiana: las intervenciones en el proceso de aprendizaje con ofertas cognoscitivas e instrumentales incide en la constitución del Yo y por ende en el posicionamiento subjetivo del niño. Los movimientos en la dirección de un tratamiento psicopedagógico facilitan un mejor pasaje desde lo idéntico a lo semejante y generan la disminución de modalidades de pensamiento viscosas de muchos niños (Levy, 1995).

\section{Denominación, definición, función interpretación}

La denominación del concepto "viscosidad del pensamiento" en los procesos cognoscitivos refiere a un observable clínico en los niños con patologías del desarrollo. Sin embargo, la autora considera que tal viscosidad también está presente, aunque en niveles menores, en los procesos de construcción cognoscitiva de los niños normales con problemas de aprendizaje, en neurosis graves en la infancia y en ciertas psicosis infantiles de diferente manera. "Una de las maneras en que se manifiesta la viscosidad en las reproducciones de lo idéntico de algunas neurosis graves en la infancia y en algunas psicosis infantiles" (Levy, 1995, p. 18). 
La definición del concepto "viscosidad del pensamiento", también llamado inercia mental, supone cierta adhesividad del pensamiento en la infancia con perseveraciones observables en distintas áreas cognoscitivas. La viscosidad del pensamiento consiste en una dificultosa tramitación del pasaje de lo idéntico a lo semejante (Levy, 1995). Desde el punto de vista de sus esquemas cognitivos, repiten las nociones aprendidas, de manera estereotipada. Se da un predominio de la acomodación, sin que puedan generalizar los esquemas de acción. La viscosidad del pensamiento hace que queden adheridos a esquemas inferiores. Al no haber cierres en las estructuras alcanzadas, regresan a modos anteriores de funcionamiento. Al coexistir diferentes niveles de pensamiento, los esquemas inferiores funcionan como polos de atracción.

Levy (1995) afirma que descubrió la viscosidad del pensamiento en las ecolalias de los niños con patologías del desarrollo. Ejemplo son niños que apagan las luces una y otra vez. Están adheridos a una presencia y ausencia que, ecopraxicamente, reproducen resoluciones infructuosas, sin intención ni posibilidad de reformularlas, ni de efectuar corte alguno.

Por último, la función interpretación del concepto "viscosidad del pensamiento" posibilita analizar en qué momento de la constitución del sujeto nos encontramos, en qué posición, con qué armado psíquico, con qué instrumentos cognoscitivos y con respecto a qué objeto de conocimiento están disponibles u obstaculizadas las funciones del Yo y la posición del sujeto. Las producciones reiterativamente viscosas, en las que fenómenos de inercia mental son un real clínico observable, deben entenderse de diferente manera según sean producto de trastornos de pensamiento menores o mayores, asociados a componentes neurológicos, lingüísticos, sensoriales, psíquicos y cognitivos con sus diferentes combinaciones. Así, la autora propone trabajar con las docentes como también ofrecer instrumentos y estrategias cognoscitivas en el tratamiento psicopedagógico para facilitar el pasaje de lo idéntico a lo semejante.

\section{Desplazamiento conceptual}

En este contexto, la autora propone el concepto de "viscosidad del pensamiento" extendiendo la noción freudiana de viscosidad libidinal con la intención de articularla con la noción piagetiana de pensamiento a la luz de observables o referentes clínicos (Levy, 1992). Si bien los procesos de equilibración tienen lugar en los distintos ámbitos cognitivos, no existe un pensamiento general cuya estructura sea aplicable a los diferentes dominios del conocimiento, ya que cada uno supone legalidades, construcciones e interacciones especificas, soportes neuropsicológicos apropiados y una particular manera de libidinizarlos (Levy, 2001). Así, dar cuenta de las perturbaciones en el pensamiento requiere de una profunda reflexión respecto a las múltiples significaciones de las producciones clínicas infantiles, con alternancias y suspensiones sucesivas, y efectuar posteriormente interpelaciones recíprocas entre las disciplinas, entre las disciplinas y prácticas clínicas que intervienen (Levy, 2001).

Por ello es necesario saber que no solo los procesos de estructuración psíquica en la infancia determinan las modalidades cognoscitivas, sino que también las estrategias cognoscitivas e instrumentales que generamos los psicopedagogos se constituyen en soportes del proceso de estructuración psíquica primaria y secundaria. En otros términos, los aportes cognoscitivos e instrumentales promueven un ensanchamiento de ciertas funciones del Yo 
(Levy, 1995). Por estos argumentos, ubicamos a 'viscosidad del pensamiento' al igual que el anterior- como un nuevo concepto teórico propio del campo psicopedagógico (Follari, 2016).

\section{Grado de rigurosidad epistemológica}

Con respecto al grado de rigurosidad de sus aportes conceptuales al campo psicopedagógico, podemos destacar que la autora, por un lado, explicita su posicionamiento epistemológico dando cuenta, en palabras de Bachelard (1987), de una actitud de vigilancia y, por otro, que es significativo su esfuerzo por distanciarse de la visión normativa prescriptiva.

También, advertir continuidades en su posicionamiento, en un sentido general, y en sus conceptualizaciones acerca de la interpelación interdisciplinar y viscosidad del pensamiento entre los encuadres culturales que recupera y los desplazamientos conceptuales que propone para las prácticas psicopedagógicas. En otras palabras, si bien incorpora aportes y va desplegando nuevas ideas, creemos que mantiene el carácter constructivo, dinámico y orientado a la novedad de los conceptos de origen.

Asimismo, observar que fundamenta su posicionamiento en una propuesta articulatoria de 'relación entre teorias' en la que, a través de la interpelación interdisciplinaria, postula operaciones de suspensión momentánea e inevitable- de algunas significaciones por efecto de centración en otra y la valida, señalando que, por estructura, es imposible realizar lecturas múltiples.

\section{CONCLUSIONES FINALES}

El análisis de la producción escrita de Evelyn Levy muestra una profunda preocupación por los procesos de aprendizaje y conocimiento de los niños. Desde los inicios de su carrera profesional, Levy se vio motivada por ciertos interrogantes que se desprendian de su práctica y formación, y que la llevaron a buscar respuestas en teorias que hasta el momento no encontraban puntos de acuerdo. Motivada por interrogantes en torno a cómo se aprende y se conoce, hallamos en su trabajo la producción de dos conceptos - los que hemos desarrollado-, que entendemos son de carácter teórico, dirigidos al campo psicopedagógico. Interesa destacar la relevancia de este aporte teórico, que se inicia en la década de los 90 , en tanto comienza a ocupar un área de vacancia y a forjar las bases de la Psicopedagogía. En la actualidad, su legado en la clínica de la escucha y la mirada psicopedagógica se reconoce en otros profesionales y en sus referentes actuales, como es el caso de Norma Filidoro, quien asume en sus producciones las fuertes contribuciones y enseñanzas adquiridas con esta autora.

Valoramos también, a partir de esta investigación, la fertilidad que tiene reconstruir encuadres culturales, conceptualizaciones así como desplazamientos conceptuales en estas producciones en términos de visibilizar los atravesamientos de nuestras prácticas profesionales y de nuestros modos de producir conceptos en pos de fortalecer la relación entre ciencia y profesión. Y también la fecundidad de recuperar -a través de las argumentaciones esgrimidas- la rigurosidad epistemológica de las mismas en aras de aportar a la constitución epistémica de la disciplina.

Finalmente, asumir el desafio en esta investigación de crear una técnica de exploración específica, ante la ausencia de recursos y tradición de este tipo 
de estudios en la psicopedagogía, y que dio lugar a una nueva contribución en términos de producción de conocimientos técnicos.

\section{REFERENCIAS}

Artacho, J. A., \& Ventura, A.C. (2011). Psicopedagogía e interdisciplina: reflexiones desde una perspectiva epistemológica. Revista Aprendizaje Hoy, 80, 7-16.

Bachelard, G. (1987). La formación del espiritu científico. México: Siglo XXI.

Bertoldi, S. (mayo, 2017). ¿Existe un 'campo científico' en la psicopedagogía argentina? Ponencia presentada en las V Jornadas de Psicopedagogía del Comahue, Viedma, Río Negro, Argentina.

Bertoldi, S. (2018). Las prácticas e investigaciones científicas en psicopedagogía en ámbitos universitarios público/estatales. Un análisis epistemológico en torno a los discursos y prácticas en la Argentina actual. 1972-2015 [Tesis Doctoral en preparación]. Facultad de Ciencias de la Educación. Universidad Nacional del Comahue, Cipolletti, Río Negro.

Bertoldi, S. \& Enrico, L. (2014-2017). PIN I: Producción de categorías en psicopedagogía y sus sustentos epistemológicos. Argentina 2014-2017. Secretaría de Ciencia y Técnica, Centro Universitario Regional Zona Atlántica, Universidad Nacional Del Comahue.

Bertoldi, S. \& Porto, M. C. (2015). Una herramienta de lectura epistemológica para analizar textos psicopedagógicos. Pilquen - Sección Psicopedagogía, 12(2), 64-71.

Bertoldi, S., \& Tovani, A. (2016). Epistemologia y Psicopedagoga. Encuadre Cultural de la producción escrita de la psicopedagoga Liliana González. Pilquen, Sección Psicopedagogía, 13(2), 10-19.

Canguilhem, G. (2009). Estudios de historia y de filosofia de las ciencias. Buenos Aires: Amorrortu Editores.

Castorina, J. A. (2016). Algunos problemas epistemológicos de la teoría psicológica y de la práctica psicopedagógica. Pilquen, Sección Psicopedagogía, 13(2), 48-62.

Enrico, L. \& Fernández, M. L. (2016). Algunas apreciaciones acerca de los conceptos de inteligencia y aprendizaje en la teorización psicopedagógica. Pilquen, Sección Psicopedagogía, 13(2), 1-9.

Fernández, A. (2000). Poner en juego el saber. Buenos Aires: Nueva Visión.

Follari, R. (2013). Epistemología, ciencias y profesiones: se hace camino al andar. Pilquen, Sección Psicopedagogía, 10, 1-7.

Follari, R. (2016). Teoría científica y práctica profesional: relaciones no lineales e imprescindibles. Pilquen, Sección Psicopedagogía, 13(2), 39-47.

Giachetti, E., \& Ventura, A. C. (2017). Contribuciones teóricas de Sara Pain a la especificidad del campo psicopedagógico: Obra e impacto en revistas nacionales. Revista de Psicología y Psicopedagogía de la Universidad del Salvador, 2, 1-30.

Levy, E. (1982). Un enfoque psicopedagógico en el trabajo clínico con niños deficientes mentales. Revista Argentina de Psicología, 31, 57-72.

Levy, E. (1987). El psicopedagogo, la interdisciplina, el conocimiento y el otro. Temas de Psicopedagogía, 3, 109-124.

Levy, E. (1988a). Reflexiones en torno a la clínica psicopedagógica y psicomotriz. En A. Jerusalinsky (Ed.), Psicoanálisis en problemas del desarrollo infantil (pp. 150-162). Buenos Aires: Nueva Visión.

Levy, E. (1988b). Análisis de los procesos de construcción de la lengua escrita en niños con síndrome de Down. Abordaje psicogenético. En A. Jerusalinsky (Ed.), Psicoanálisis en problemas del desarrollo infantil (pp. 265-288)

Levy, E. (1990). Sujeto y objeto en la clínica psicopedagógica. Su presencia y posición. Temas de Psicopedagogía, 4, 47-63.

Levy, E. (1991). Posición e intervención en la dirección del tratamiento psicopedagógico. Temas de Psicopedagogía, 5, 43-57.

Levy, E. (1992). El proceso diagnóstico en la intervención psicopedagógica. Aprendizaje Hoy, 23/24, 7-13.

Levy, E. (1994). De la significación a la intervención en la clínica psicopedagógica hospitalaria. Temas de Psicopedagogía, 6, 195-205.

Levy, E. (1995). Viscosidad de pensamiento y procesos cognoscitivos. Una interpelación interdisciplinaria. Escritos de la Infancia, 6, 15-33.

Levy, E. (1996). A interpelação interdisciplinar na clínica psicopedagógica. Estilos Da Clínica, 1(1), 128-145. https://doi.org/10.11606/issn.1981-1624.v1i1p128-145

Levy, E. (2001). Posibles implicancias reciprocas entre Psicología Genética y Clínica Psicopedagógica. Estrategias y procedimientos clínicos. En J. A. Castorina (Eds.), Desarrollos y Problemas en Psicología Genética (pp. 291-312). Eudeba: Buenos Aires.

Ortiz, E., \& Mariño, M. (2014). Una comprensión epistemológica de la psicopedagogía. Cinta de moebio, 49, 22-30. https://doi.org/10.4067/S0717-554X2014000100003

Recibido 07-04-2018 | Aceptado 19-10-2018

Este trabajo se encuentra bajo una Licencia Creative Commons Atribución 4.0 Internacional que permite a terceros utilizar lo publicado siempre que se dé el crédito pertinente a los autores y a Psicodebate 